\title{
RHIC Operations with asymmetric collisions in 2015
}

C. Liu, E. C. Aschenauer, G. Atoian, M. Blaskiewicz, K. A. Brown, D. Bruno, R. Connolly, T. D. Ottavio, K. A. Drees, W. Fischer, C. J. Gardner, X. Gu, T. Hayes, H. Huang, J. S. Laster, Y. Luo, Y. Makdisi, G. Marr, A. Marusic, F. Meot, K. Mernick, R. Michnoff, M. Minty, C. Montag, J. Morris, G. Narayan, S. Nayak, S. Nemesure, P. Pile, A. Poblaguev, V. Ranjbar, G. Robert-Demolaize, T. Roser, B. Schmidke, V. Schoefer, F. Severino, T. Shrey, K. Smith, D. Steski, S. Tepikian, D. Trbojevic, N. Tsoupas, G. Wang, S. White, K. Yip, A. Zaltsman, K. Zeno, S. Y. Zhang

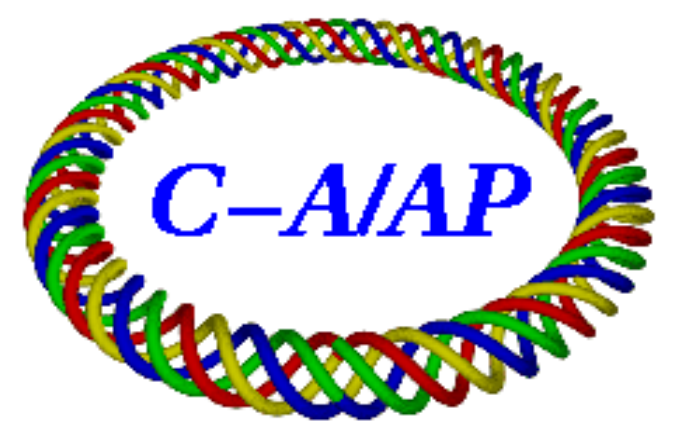

Collider-Accelerator Department Brookhaven National Laboratory Upton, NY 11973

\section{U.S. Department of Energy}

Office of Science, Office of Nuclear Physics

Notice: This document has been authorized by employees of Brookhaven Science Associates, LLC under Contract No. DE-SC0012704 with the U.S. Department of Energy. The United States Government retains a nonexclusive, paid-up, irrevocable, world-wide license to publish or reproduce the published form of this document, or allow others to do so, for United States Government purposes. 


\title{
RHIC OPERATION WITH ASYMMETRIC COLLISIONS IN 2015*
}

\author{
C. Liu, E. C. Aschenauer, G. Atoian, M. Blaskiewicz, K. A. Brown, \\ D. Bruno,R. Connolly, T. D. Ottavio, K. A. Drees, \\ W. Fischer, C. J. Gardner, X. Gu, T. Hayes, H. Huang, \\ J. S. Laster, Y. Luo, Y. Makdisi, G. Marr, A. Marusic, \\ F. Meot, K. Mernick, R. Michnoff, M. Minty, C. Montag, J. Morris, \\ G. Narayan, S. Nayak, S. Nemesure, P. Pile, A. Poblaguev, V. Ranjbar, \\ G. Robert-Demolaize, T. Roser, B. Schmidke, V. Schoefer, F. Severino, \\ T. Shrey, K. Smith, D. Steski, S. Tepikian, D. trbojevic, N. Tsoupas, \\ G. Wang, S. White, K. Yip, A. Zaltsman, K. Zeno, S. Y. Zhang, \\ Brookhaven National Lab, Upton, NY, U.S.A.
}

August 7, 2015

\begin{abstract}
To study low-x shadowing/saturation physics as well as other nuclear effects 1, 2, proton-gold (p-Au, for 5 weeks) and proton-Aluminum (p$\mathrm{Al}$, for 2 weeks) collisions were provided for experiments in 2015 at the Relativistic Heavy Ion Collider (RHIC), with polarized proton beam in the Blue ring and $\mathrm{Au} / \mathrm{Al}$ beam in the Yellow ring. The special features of the asymmetric run in 2015 will be introduced. The operation experience will be reviewed as well in the report.
\end{abstract}

\section{Introduction}

The revolution frequencies of two asymmetric beams need to be matched both at store and during acceleration at RHIC [3, 4, 5]. This requires a close energy per nucleon for both beams. However, the beam rigidities differ by a factor of 2.5 for $\mathrm{p}-\mathrm{Au}$. The consequence is that the orbits and acceleration cycle for $\mathrm{p}-\mathrm{Au} / \mathrm{Al}$ collision were different from symmetric species collision.

The orbits of both beams shift towards the Yellow beam line horizontally in the interaction regions (IRs). RHIC is a collider with two nearly independent rings and DX magnets, which combine two beams at the IRs, as the only common dipole magnet. The orbits (Fig. 1) shift horizontally when two beams with

*The work was performed under Contract No. DE-SC0012704 with the U.S. Department of Energy. 
unequal rigidities going through a common dipole magnet. As the result, the DX magnets and other components need to be moved to make enough aperture for the beams [6. There are more details on beam orbits at injection, at store, for colliding and non-colliding IRs in reference [7.

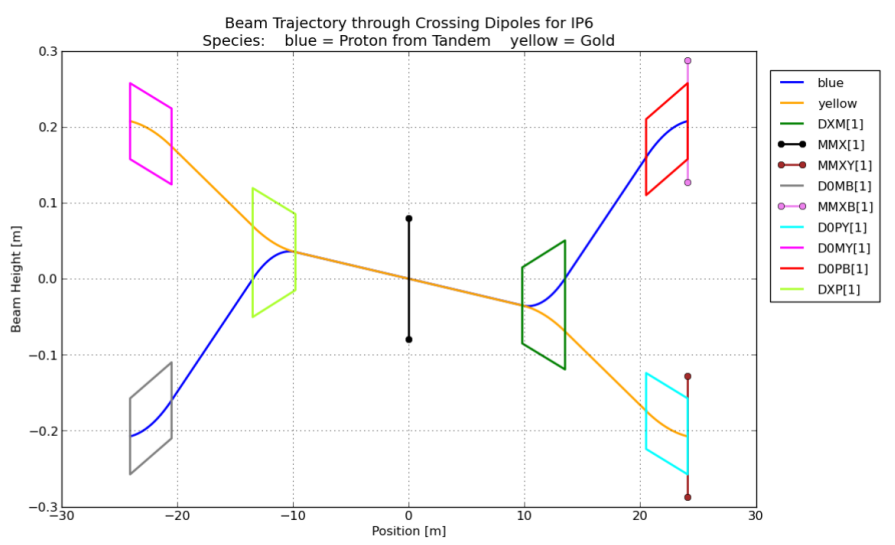

Figure 1: The orbits at STAR experiment in p-Au run. The Blue and Yellow beams collide head-on with a common angle of $3.62 \mathrm{mrad}$. The two magnets closer to IP $(0 \mathrm{~m})$ are the common DX magnets. The other four are the D0 magnets.

The ramp is designed as two parts to be able to match the frequencies of two beams as shown in Fig. 2. All species are injected into RHIC with more or less the same beam rigidity for high energy collisions. The Au beam injection energy can't be raised enough to match that of proton beam due to the limit of the injection kicker strength (86 Tm). It is unfavorable to lower the proton beam injection energy below transition ( $22 \mathrm{GeV}$ ) to match that of gold beam. Therefore, the gold beam is accelerated to an intermediate energy, then proton beam is injected with matched frequencies and both beams are accelerated to top energies. The first ramp was tested in 2014 with a conclusion that there is no instability issue of Au beam sitting close to transition [8].

The proton beam energy was chosen for the required center-of-mass energy. Proton beam energy at store is usually set at $G \gamma$ equals half integer for better polarization lifetime. Au beam energy is therefore determined to match beam frequencies. The center-of-mass energy of $\mathrm{p}-\mathrm{Au}$ is required to be close to that of p-p in $2015(200 \mathrm{GeV} / \mathrm{n})$. If the proton energy was kept at $100 \mathrm{GeV} / \mathrm{n}$ $(G \gamma=191.5)$, the center-of-mass energy of $\mathrm{p}-\mathrm{Au}$ would be $195 \mathrm{GeV} / \mathrm{n}$. To have the center-of-mass energy of p-Au as $200.6 \mathrm{GeV} / \mathrm{n}$, which is as close to $200 \mathrm{GeV} / \mathrm{n}$ as can be, the $G \gamma$ would be 197.5. In the end, the proton $G \gamma$ was set to 187.5 where there was no strong intrinsic depolarization resonances nearby(Fig. 3) and the center-of-mass energy $(201.6 \mathrm{GeV} / \mathrm{n})$ was close enough to $200 \mathrm{GeV} / \mathrm{n}$. 


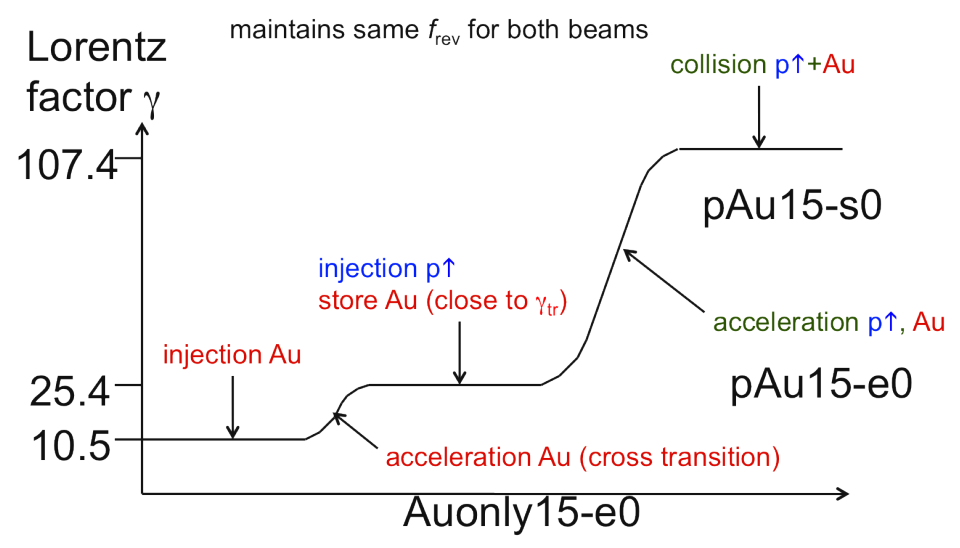

Figure 2: The acceleration cycle for $\mathrm{p}-\mathrm{Au}$. The $\mathrm{Au}$ beam is injected at $\gamma=10.5$. It is accelerated above transition to $\gamma=25.4$ during a mini-ramp (Auonly15$\mathrm{e} 0$ ). While the $\mathrm{Au}$ beam sits on the porch, the polarized proton beam is injected with matched frequency. Then both beams are accelerated (pAu15-e0) to top energies.

\section{Operations review}

\subsection{Operations overview}

The operation was more complicated compared than previous runs due to the special ramp design. The operators needed to tune proton beam in the injectors to make sure transmission, polarization and other parameters are acceptable. Then the operation mode will be switched to gold, which involves ramping down cold snake in AGS (6 mins), tuning gold beam in the injectors and so on. With some tuning in RHIC, gold beam was filled in the Yellow ring and accelerated to cross transition. At the same time the operation mode will be switched back to proton in the injectors which ramps up the cold snake. Tuning of proton beam in RHIC was under time pressure because beam emittance dilution of Au beam due to intra-beam scattering (IBS) is expected while it sits on the porch just above transition. Then proton beam was filled in the Blue ring and both beams were accelerated to top energies. With the automation of mode-switching, the operators were able to keep the turn-around time between 7-hour stores on the same level as a symmetric run. The average was $1.74 \mathrm{hrs}$ in p-Au (1.18 \& 3.75 hrs for $25 \mathrm{th} \% \& 75 \operatorname{th} \%), 1.82 \mathrm{hrs}$ in p-Al (1.22 \& $2.58 \mathrm{hrs}$ for $25 \operatorname{th} \% \& 75 \operatorname{th} \%)$.

\subsection{Beam emittance}

The beam emittance of both beams are critical for not only the delivered luminosity but also for clean background at experiments. Because the proton bunch intensity was two orders of magnitude larger than the Au intensity, stochastic cooling [9] was only able to operate in the Yellow ring for gold beam at store. In 


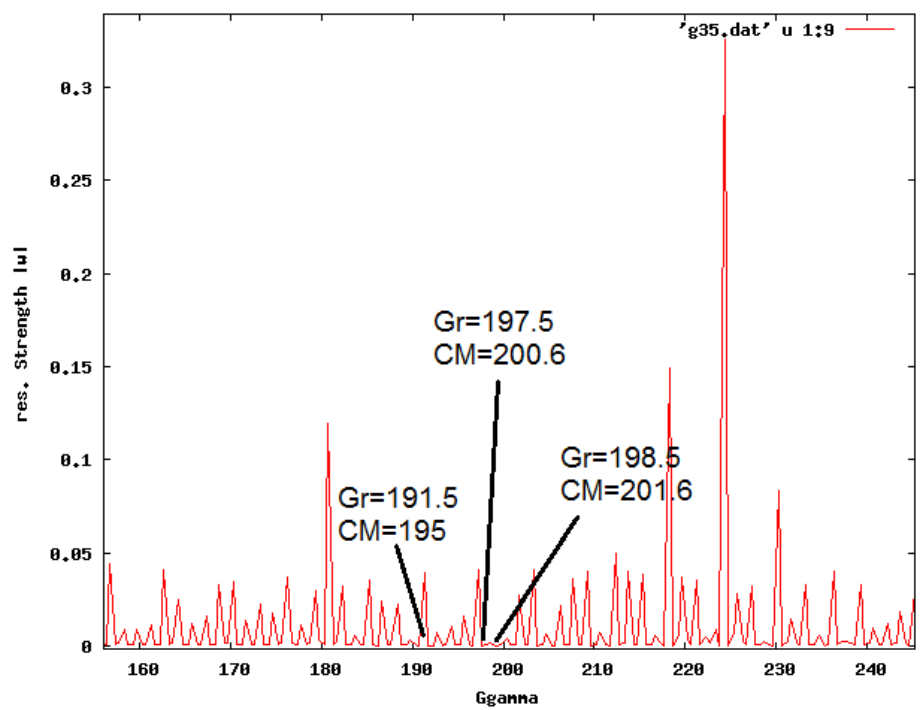

Figure 3: Intrinsic depolarization resonance nearby $100 \mathrm{GeV}$ proton.

the first half of p-Au run, the proton beam loss rate showed strong dependency on the cooling speed. The cooling was set with the full strength for $\sim 3 \mathrm{hrs}$ guided by simulations [10, 11] and operational experience. Then the strength was reduced to keep emittance of gold beam constant afterwards (Fig. 44. The goal was to maximize the integrated luminosity and keep the background at STAR from the proton beam loss acceptable. For the second half of the run, proton beam loss was much reduced with a new working point at injection, so that the cooling was on with full strength for the majority part of a store until the proton beam loss approached $10 \%$ (Fig. 4 .
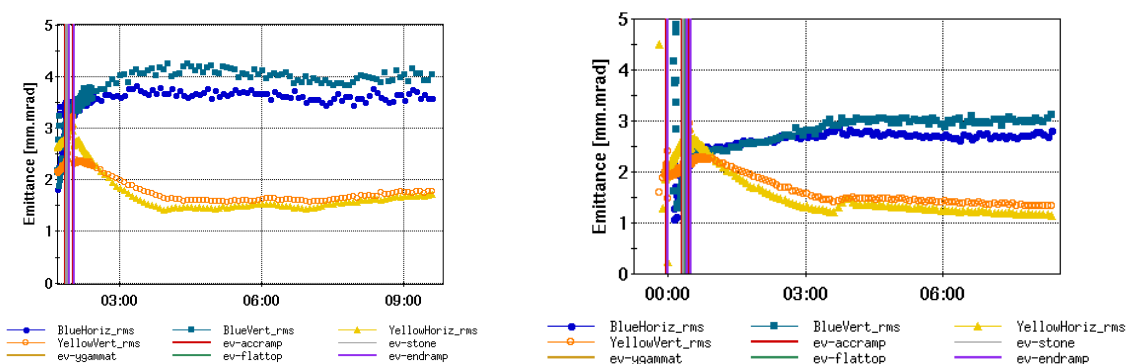

(a) Beam emittances at store during the(b) Beam emittances at store during the first half of p-Au run. second half of p-Au run.

Figure 4: Evolution of beam emittances and stochastic cooling configuration.

While the gold beam stays at the intermediat energy on the porch for $\sim 30$ 
minutes, beam emittance dilutes due to IBS effect. The dilution of emittance was in agreement with the simulation results (Fig. 5) during the first ramp, on the porch and during the second ramp. The top plot shows the bunch length reported by wall current monitor (WCM). The bunch shape was distorted by the Landau cavity. At the end the bunch length is $6.4 \mathrm{~ns}$, whereas the simulated bunch length is $5.7 \mathrm{~ns}$. The middle plot is the longitudinal emittance derived from the bunch length. At the end, the measured emittance from WCM was $1.24 \mathrm{eVs}$, whereas the simulated emittance was $0.97 \mathrm{eVs}$. The IBS longitudinal emittance growth on the long porch is weaker than the second ramp with higher energy. The bottom is the transverse emittance. It started from $10.5 \mu \mathrm{m}$, and ended up with $17.8 \mu \mathrm{m}$. The vertical emittance reported by IPM caught up during the late part of the second ramp possibly due to linear coupling.

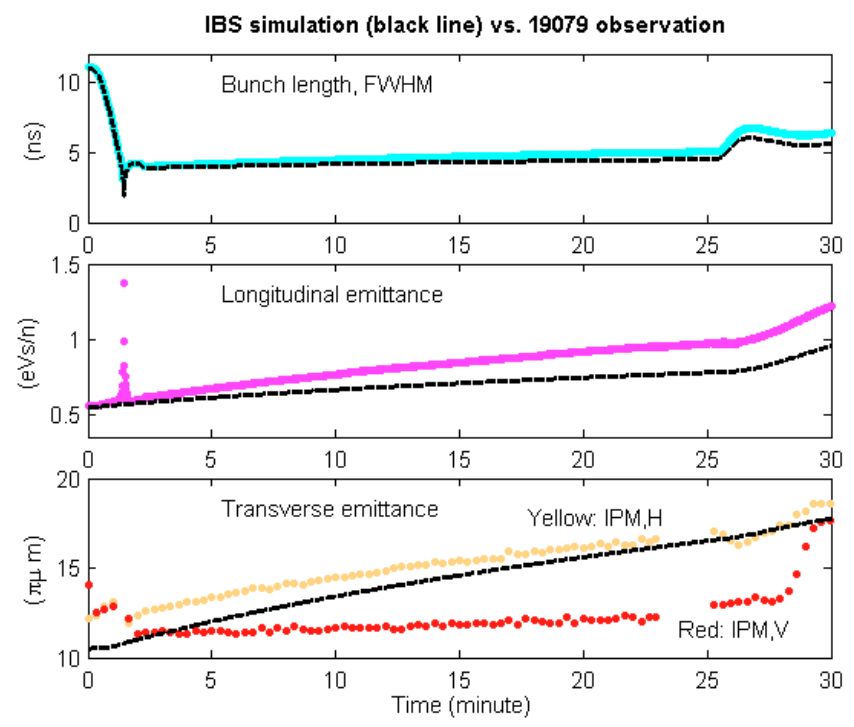

Figure 5: Intra-beam scattering simulation for Au beam during the Auonly15-e0 ramp, at the porch and the pAu15-e ramp. The first acceleration started at 0 minutes, the second acceleration started around 27 minutes.

At the beginning of the run, the working point for proton beam was set to be $(0.6926,0.6898)$. It was reverted back to the nominal working point at $(0.695,0.692)$. The beam loss rate at injection was reduced from $100 \% / \mathrm{h}$ down to $20 \% / \mathrm{h}$. Loss rate on the ramp was reduced as well. The proton beam emittance was reduced at injection, during acceleration and at store. The peak collision rate increased from mid $20 \mathrm{~s} \mathrm{kHz}$ to mid $30 \mathrm{~s} \mathrm{kHz}$ with all other conditions identical. 


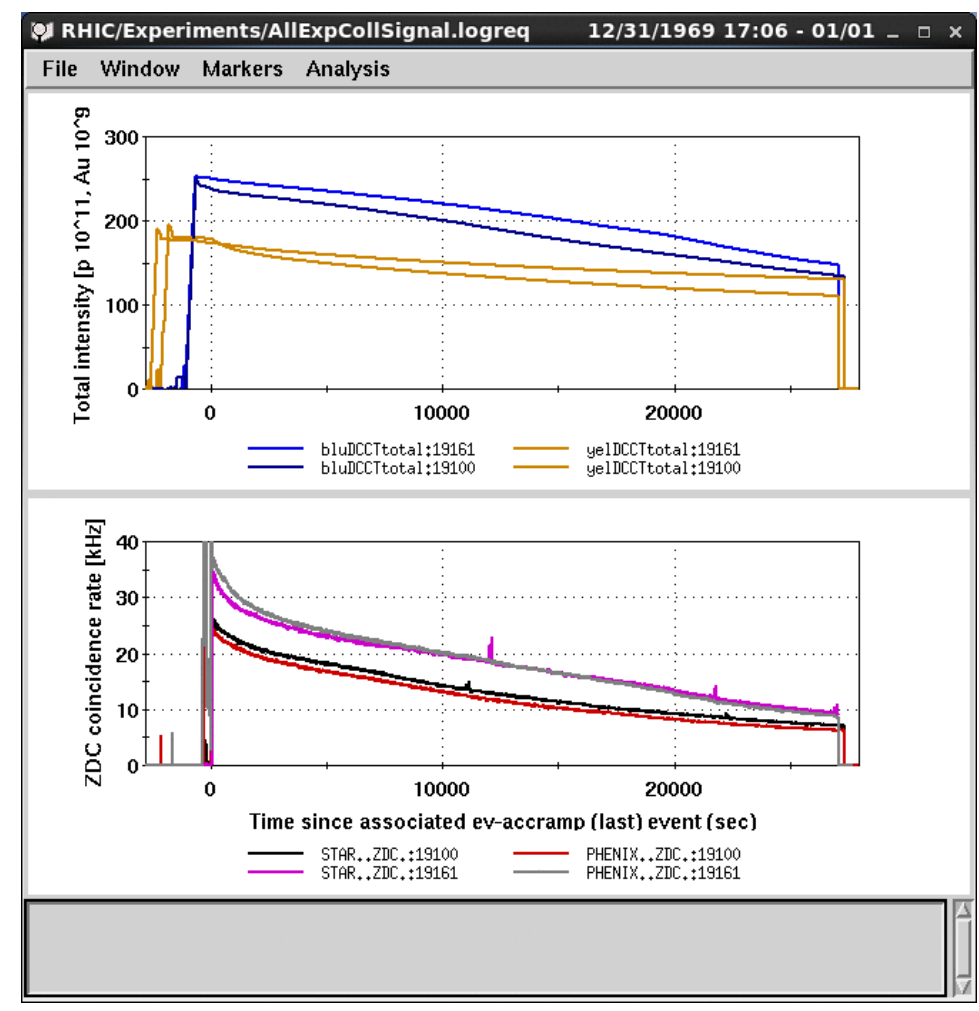

Figure 6: The impact of proton injection working point on the experiments collision rates. The beam intensities for fill 19100 and 19161 are the same. The working point for fill 19161 was at nominal $(0.695,0.692)$. The working point for fill 19100 was at $(0.6926,0.6898)$.

\subsection{Beam intensity}

The intensity of proton beam was improved by employing the second harmonic cavity in the Booster. To combat space charge effect at Booster injection, the second harmonic cavity was phased 180 degrees from the main cavity to lengthen the bunch 12 . As the result, the transverse emittance in AGS and RHIC were reduced by $20 \%$. So the proton intensity can be improved to $2.5 \times 10^{11}$ with beam polarization in the upper 50 s percent.

Neither of the proton or gold beam intensity provided by the injectors was beam-beam limited in RHIC [5]. The intensity would be $4 \times 10^{11}$ for proton and $4 \times 10^{9}$ for Au to reach beam-beam limit, which is the same as the beambeam parameter for $1.6 \times 10^{11} \mathrm{pp}$ run. Major beam loss for the Au beam is at transition. The ramp rate at transition for the mini-ramp is slower than that of Au-Au ramp. With tuning, the transmission efficiency (93\%) was just a little lower than Au-Au (95\%). A 6-3-1 merge scheme in AGS was successfully implemented [13. The bunches with $20 \%$ higher intensity were ramped in 
RHIC. The test ramp with 12 bunches was successful. The ramp with full machine was aborted due to too much de-bunched beam. Intensity dependency of the polarization were observed both in the injectors and RHIC. Therefore, proton beam intensity was set at $2.3 \times 10^{11}$ ppb for most part of the asymmetric run to keep store polarization around $60 \%$. Al intensity provided by the injectors was higher than what RHIC can take in 2015 . With $>1.3 \times 10^{10} \mathrm{ppb}$, loss at transition crossing was too much, and there was difficulty associated with rebucketing.

\subsection{Lattice}

For $100 \mathrm{GeV}$ p-p run in 2015, a new lattice 14 was designed with a multiple of 180 degrees phase advance from e-lens [15] to IP8. Even though it was not necessary to compensate beam-beam using e-lens, the same lattice was used in the proton ring for $\mathrm{p}-\mathrm{Au} / \mathrm{Al}$ run because better dynamic aperture performance demonstrated during p-p run. The lattice for $\mathrm{Au}$ beam in $\mathrm{p}-\mathrm{Au} / \mathrm{Al}$ was the same as in $2014100 \mathrm{GeV} \mathrm{Au}-\mathrm{Au}$ run.

\section{Performance review}

The beam polarization of the asymmetric run measured by p-Carbon [16] and $\mathrm{H}$-jet polarimeters 17 are shown in Figs. 7 and 8 . The average polarization at store were $60 \%$ and $53 \%$ for $\mathrm{p}-\mathrm{Au}$ and $\mathrm{p}-\mathrm{Al}$ run measured by $\mathrm{p}$-Carbon. The average polarization at store were $60 \%$ and $54 \%$ for $\mathrm{p}-\mathrm{Au}$ and $\mathrm{p}-\mathrm{Al}$ run measured by H-jet.

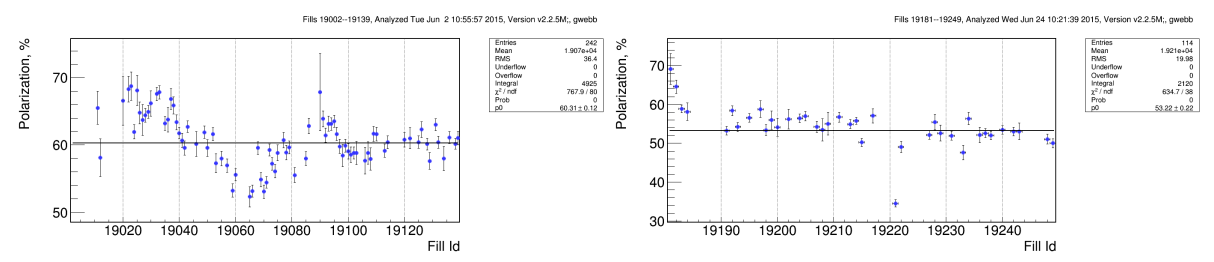

(a) Beam polarization measured by $\mathrm{p}$-(b) Beam polarization measured by $\mathrm{p}$ Carbon polarimeter during p-Au run. Carbon polarimeter during p-Al run.

Figure 7: p-Carbon polarization during Run15.

The integrated luminosities of $\mathrm{p}-\mathrm{Au} / \mathrm{Al}$ run are shown in Figs. 9 and 10 Due to a STAR magnet transformer over-heating, there was no data taking for the last 3 days in STAR. Without the STAR solenoidal magnet, electron clouds can form inside the experiment beam pipe. To keep the pressure rise due to secondary electron emission under $10^{-7}$ Torr, the beam intensity was kept at $2 \times 10^{11} \mathrm{ppb}$ for proton and $1 \times 10^{10} \mathrm{ppb}$ for $\mathrm{Al}$. 

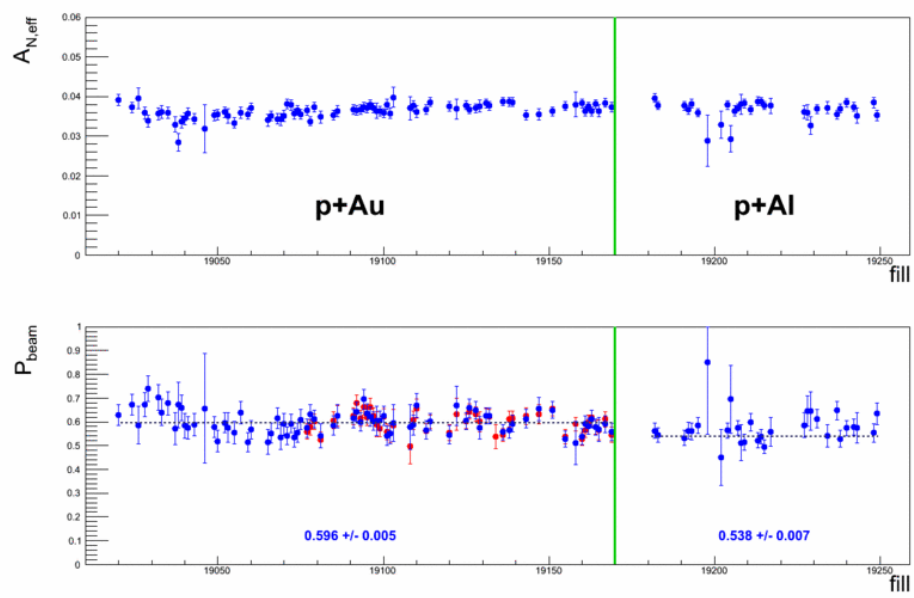

Figure 8: Beam polarization measured $\mathrm{H}$-jet polarimeter during $\mathrm{p}-\mathrm{Au} / \mathrm{Al}$ run.

\section{New developments}

To combat longitudinal diffusion at store, a superconducting $56 \mathrm{MHz}$ cavity was designed as a common component for both beams [18, 19. The tests and operation in 2014 was limited by the heating of the HOM couplers. Beam study in 2015 without HOMs showed HOM mode damping is critical for cavity operation. The cavity frequency sweeps through first four monopole HOM resonance lines within the full tuning range. Beam is affected greatly by the excited HOMs in the cavity. During p-Au operation, HOM modes excited by off-center proton beam blows up the longitudinal emittance. Solution was to use the fundamental power coupler (FPC) as a HOM damper.

Even though the intensity of proton beam was not beam-beam limited, the fact that the tunes $(0.6884 / 0.6834$ for proton, 0.228/0.226 for $\mathrm{Au} / \mathrm{Al})$ are far away for $\mathrm{p}-\mathrm{Au} / \mathrm{Al}$ provided a unique chances for beam-beam compensation study with e-lens 15 due to the absence of coherent beam-beam modes. With reduced proton beam angle, eletron beam was aligned to the proton beam, and tune spread compression due to the electron lens could be measured with beam transfer functions.

To combat the possible beam instability with e-lens solenoid, transverse bunch-by-bunch dampers were designed for both beams in both planes. Beam tests were done successfully except for Yellow horizontal due to hardware issue. However, excessive beam loss was observed while trying to engage the feedback for operation. 


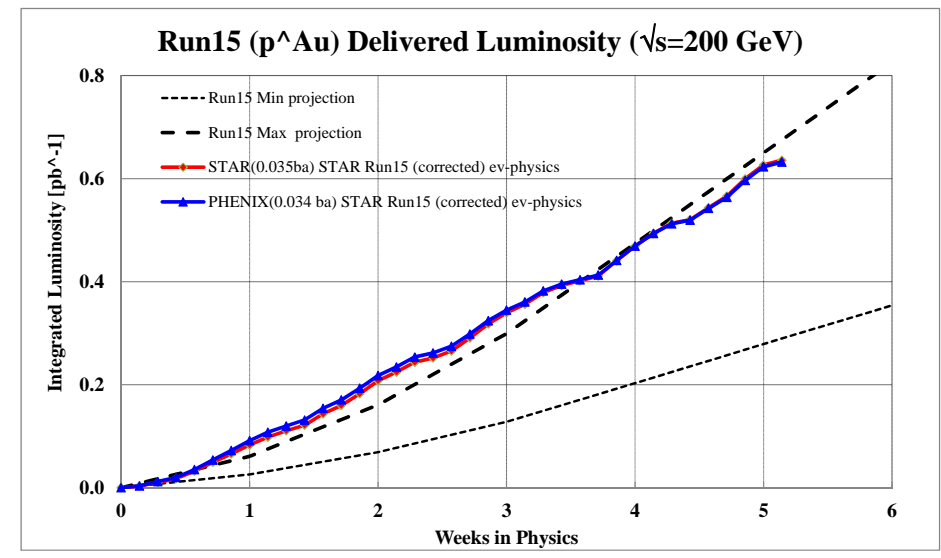

Figure 9: Delivered luminosity during the 5 weeks p-Au run.

\section{Summary}

RHIC has demonstrated p-Au and p-Al collisions in 2015. The collision of proton and even heavier ions than gold is possible with the same machine configurations for $\mathrm{p}-\mathrm{Au}$ collision. The operational experience of $\mathrm{p}-\mathrm{Au}$ and $\mathrm{p}-\mathrm{Al}$ special ramp is unique. The 1.75 -hr turn-around time between stores was realized by quick mode switching of the injectors and professionalism of the operators. Special runs for PHENIX polarimeter measurements with proton beam aiming at the center of ZDC were successful and generated more interests. The switching from p-Au to p-Al run took only $16 \mathrm{hrs,} \mathrm{quickest} \mathrm{in} \mathrm{RHIC} \mathrm{history.}$

\section{References}

[1] PHENIX Beam Use Proposal Run-15 and Run-16. https://indico.bnl. gov/getFile.py/access?resId=0\&materialId=0\&conf $I d=764$.

[2] STAR Beam Use Proposal For Runs 15 and 16. https://indico.bnl. gov/getFile.py/access?resId=0\&materialId=1\&conf Id=764.

[3] T. Satogata, L. Ahrens, M. Bai, J. Beebe-Wang, M. Blaskiewicz, JM. Brennan, KA. Brown, D. Bruno, PP. Cameron, J. Cardona, et al. Commissioning of RHIC deuteron-gold collisions. In Particle Accelerator Conference, 2003. PAC 2003. Proceedings of the, volume 3, pages 1706-1708. IEEE, 2003. 


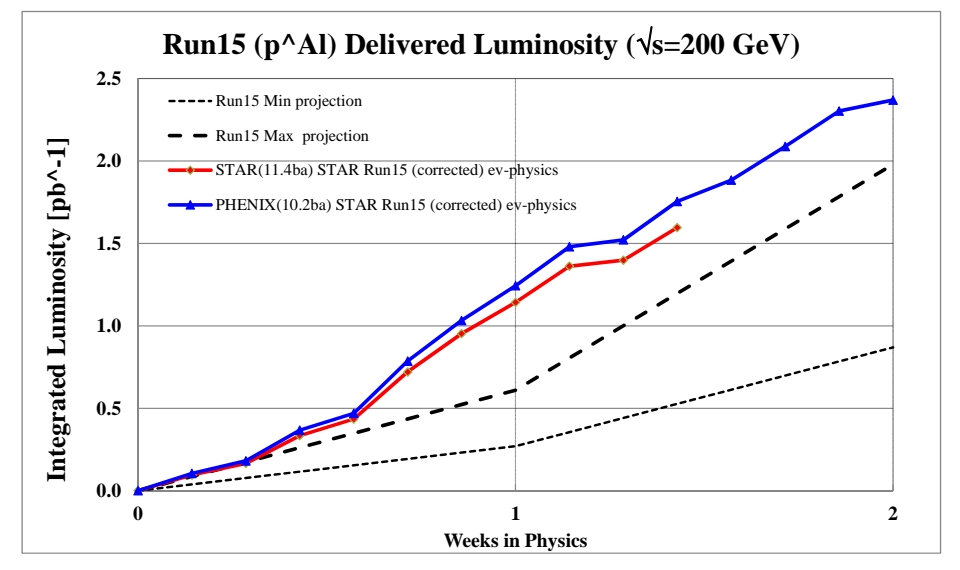

Figure 10: Delivered luminosity during the 2 weeks p-Al run.

[4] W. Fischer, P. Cameron, S. Peggs, and T. Satogata. Tune modulation from beam-beam interaction and unequal radio frequencies in RHIC. In AIP Conference Proceedings, pages 252-255, 2003.

[5] C. Liu. Attempt to accelerate asymmetric species with unequal frequencies in RHIC. Technical report, C-A/AP/5xx, 2015.

[6] C. Liu. Preparations for p-Au run in 2015. Technical report, C-A/AP/532, 2014.

[7] FY15 Run Planning To Do List. http://www.cadops.bnl.gov/AGS/ Operations/OpsWiki/index.php/FY15_Run_Planning_To_Do_List.

[8] C. Montag, M. Blaskiewicz, V. Schoefer, I. Blackler, A. Marusic, and T. Shrey. p-Au ramp test. Technical report, C-A/AP/518, 2014.

[9] M. Blaskiewicz, JM. Brennan, and K. Mernick. Three-dimensional stochastic cooling in the Relativistic Heavy Ion Collider. Physical review letters, 105(9):094801, 2010.

[10] K. Mernick. http://www.cadops.bnl.gov/cgi-bin/elog/viewMain. pl?elog=StochasticCooling_stay\&shiftlog=Wed_Jan_7_2015_11:01: 05_AM\#20150419142612.

[11] C. Liu. http://www.cadops.bnl.gov/cgi-bin/elog/viewMain. pl?elog=rhic-pAu_2015\&shiftlog=Mon_May_18_2015_20:36: 00_PM\#20150419111336. 
[12] V. Schoefer, EC. Aschenauer, G. Atoian, M. Blaskiewicz, KA. Brown, D. Bruno, R. Connolly, T. D Ottavio, KA. Drees, Y. Dutheil, et al. RHIC polarized proton-proton operation at $100 \mathrm{GeV}$ in Run 15 . Technical report, Brookhaven National Laboratory (BNL), 2015.

[13] C. Gardner. Heavy Ion Intensity in Run 16. Presentation at RHIC retreat, 2015.

[14] S. White, W. Fischer, and Y. Luo. Optics solutions for pp operation with electron lenses at $100 \mathrm{GeV}$. Technical report, C-A/AP/519, 2014.

[15] X. Gu, Z. Altinbas, D. Bruno, S. Binello, M. Costanzo, A. Drees, W. Fischer, DM. Gassner, J. Hock, K. Hock, et al. RHIC electron lenses upgrades. IPAC 15 .

[16] RHIC Polarimetry Results: p-Carbon Measurement Database. http:// www.phy.bnl.gov/cnipol/rundb/.

[17] H-jet Polarimetry. https://wiki.bnl.gov/rhicspin/Polarimetry/ H-jet/Run15pA.

[18] AV. Fedotov and I. Ben-Zvi. Beam dynamics and expected RHIC performance with 56MHz RF upgrade. Technical report, C-A/AP/333, 2009.

[19] Q. Wu and I. Ben-Zvi. Optimization of Higher Order Mode Dampers in the $56 \mathrm{MHz}$ SRF Cavity for RHIC. IPAC10, Kyoto, Japan, 2010. 